\title{
Modeling Neuromorphic Persistent Firing Networks
}

\author{
Ning Ning ${ }^{*}$, Guoqi Li ${ }^{2,3}{ }^{*}$, Wei He${ }^{1}$, Kejie Huang4, Li Pan1, Kiruthika Ramanathan1, \\ Rong Zhao ${ }^{4}$, Luping Shi2,3 \\ ${ }^{1}$ Data Storage Institute, Agency for Science, Technology and Research, Singapore \\ ${ }^{2}$ Department of Precision Instrument, Tsinghua University, Beijing, China \\ ${ }^{3}$ Center for Brain-Inspired Computing Research (CBICR), Tsinghua University, Beijing, China \\ ${ }^{4}$ Singapore University of Technology and Design, Dover, Singapore \\ Email: Ipshi@tsinghua.edu.cn
}

Received 5 January 2015; accepted 23 January 2015; published 30 January 2015

Copyright (C) 2015 by authors and Scientific Research Publishing Inc.

This work is licensed under the Creative Commons Attribution International License (CC BY). http://creativecommons.org/licenses/by/4.0/

(c) (;) Open Access

\begin{abstract}
Neurons are believed to be the brain computational engines of the brain. A recent discovery in neurophysiology reveals that interneurons can slowly integrate spiking, share the output across a coupled network of axons and respond with persistent firing even in the absence of input to the soma or dendrites, which has not been understood and could be very important for exploring the mechanism of human cognition. The conventional models are incapable of simulating the important newly-discovered phenomenon of persistent firing induced by axonal slow integration. In this paper, we propose a computationally efficient model of neurons through modeling the axon as a slow leaky integrator, which captures almost all-known neural behaviors. The model controls the switching of axonal firing dynamics between passive conduction mode and persistent firing mode. The interplay between the axonal integrated potential and its multiple thresholds in axon precisely determines the persistent firing dynamics of neurons. We also present a persistent firing polychronous spiking network which exhibits asynchronous dynamics indicating that this computationally efficient model is not only bio-plausible, but also suitable for large scale spiking network simulations. The implications of this network and the analog circuit design for exploring the relationship between working memory and persistent firing enable developing a spiking networkbased memory and bio-inspired computer systems.
\end{abstract}

\section{Keywords}

Neuron Model, Neuromorphic, Persistent Firing, Slow Integration, Spiking Network, Working Memory

\footnotetext{
${ }^{*}$ The authors contribute equally to this work.

This work is funded by Brain Inspired Computing Research, Tsinghua University (20141080934).
} 


\section{Introduction}

Understanding how human brain represents, stores, and processes information is one of the greatest unsolved mysteries and fundamental challenges of science today. Over the past century, since Lapicque introduced the integrate-and-fire model of the neuron in 1907 [1], computational neuroscientists have developed several mathematical and computational neural models. Generally, one approach in computational neuroscience involves creating biologically realistic models, where information about the biological details of neurons including their electrochemistry, biochemistry, and detailed morphology and connectivity are also included [2], such as Hodgkin-Huxley [3] and compartment models [4]. Another approach involves building qualitative models to capture the spiking nature and the essential elements of the behavior with simplified complexity, for example, leaky integrate-and-fire [5], FitzHugh-Nagumo [6], Morris-Lecar [7], Hindmarsh-Rose [8], Wilson [9], Resonate-andFire [10] and Izhikevich [11] neuron models.

However, as neuroscience continues to advance rapidly, more and more complex neuronal behaviors and brain dynamics are revealed. This has posed challenges for neural modeling as conventional neuron models fail to reflect the newly-discovered complexity of neural systems, such as the persistent firing phenomenon recently observed in rodent hippocampal inhibitory neurons [12]. In the classic viewpoint about the information flow in the nervous system, synaptic inputs are received and integrated in the dendrites only on a timescale of milliseconds to seconds, and when the depolarized somatic membrane potential exceeds the threshold, action potentials are triggered at the axon hillock and propagate along the axon. However, recent discovery reveals that some action potential began at the distal end of axon instead of at the axon hillock. A much slower form of potential integration is observed which leads to persistent firing in distal axons of rodent hippocampal and neocortical interneurons [12]. The slow integration lasts from tens of seconds to minutes in distal axon, so does the persistent firing. During the persistent firing, the somatic depolarization is not observed, implying that axon may perform its own neural computations without any involvement of the soma or dendrites.

In this paper, we propose a new computationally-efficient artificial neuron model that account for all-known neural behaviors. In this model, axon is an independent computational unit complementary to the classic somatic computational unit which evokes action potentials. Compared to the soma which integrates dendritic inputs in a timescale of milliseconds to seconds, the axon integrates the spikes evoked by soma in a timescale of tens of second to minutes, and consequently determines the persistent firing behavior of the axon. Besides the computational model, a neuromorphic model of persistent firing neurons and its analog circuit are also proposed. In addition, a polychronous spiking network [13] with persistent firing inhibitory interneurons is simulated, which may assist the development of spiking network-based memory and bio-inspired computer system.

\section{Persistent Firing Neuron Model}

The conventional models such as Izhikevich neuron model [11] are incapable of simulating the important newlydiscovered phenomenon of persistent firing induced by axonal slow integration, as a recent discovery in neurophysiology reveals that interneurons can slowly integrate spiking, share the output across a coupled network of axons and respond with persistent firing even in the absence of input to the soma or dendrites. To this end, we extend the Izhikevich neuron model by modeling the axonal slow integration, which can be mathematically described by Equations (1)-(6):

$$
\begin{gathered}
v^{\prime}=0.04 v^{2}+5 v+140-u+I \\
u^{\prime}=a(b v-u) \\
w^{\prime}=-f w
\end{gathered}
$$

with the auxiliary resettings:

$$
\begin{gathered}
\text { if } v \geq 30 \mathrm{mV} \text {, then } v \leftarrow c, u \leftarrow u+d, w \leftarrow w+e \\
\text { if } w \geq w_{p} \text {, then }(a, b, c, d, e) \leftarrow(a, b, c, d, e)_{p} \\
\text { if } w \leq w_{n} \text {, then }(a, b, c, d, e) \leftarrow(a, b, c, d, e)_{n}
\end{gathered}
$$

where ' $=\mathrm{d} / \mathrm{dt}, t$ is the time, $w$ describes the hyper theoretical potential of the axonal leaky integrator, $v$ 
and $u$ are the dimensionless variables that describe the membrane potential, the membrane recovery, respectively. Parameters $a, b, c, d, e$ and $f$ are dimensionless, $a$ describes the timescale of $u, b$ represents the sensitivity of $u$ to the subthreshold fluctuations of $v, c$ is the after-spike reset value of the membrane potential, $d$ describes the after-spike reset of $u, e$ represents the after-somatic-spike axonal accumulation in the passive conduction mode, and $f$ is the value describing the rate of the axonal leak. In Equations (5) and (6), $w_{p}$ is the high threshold (rising edge) value of $w$ to trigger the persistent firing mode of axon, and $w_{n}$ is the low threshold (falling edge) value of $w$ for the axon to return to passive conduction mode. $(a, b, c, d, e)_{p}$ and $(a, b, c, d, e)_{n}$ describe the parameter sets of $a, b, c, d, e$ variables when the axon is in the persistent firing mode and passive conduction mode, respectively.

This model extends the Izhikevich neuron model [11] described by Equations (1)-(2), which well captures the somatic spiking dynamics. By modifying the $a, b, c, d$ variables in Equations (1), (2), and (4), different firing patterns can be generated. One important concept of our model is the axon-dependent switch of the axonal firing patterns through dynamically selecting different variable set of $a, b, c$ and $d$.

The axon is modeled as a slow leaky integrator, which is capable to alter the axonal functions between passive conduction and persistent firing modes, depending on the potential of axonal integrator. In the passive conduction mode, the axon acts as a transmission cable in which stable propagation occurs once an action potential is evoked by synaptic inputs. In the persistent firing mode, in which the parameters in the model are chosen to set the dynamical system to be self-oscillatory, axon acts as a bistable oscillator which does not require stimulus from dendrites to sustain the persistent firing of action potentials.

In contrast to the somatic leaky integrator which accounts for the integration of dendritic inputs, the axonal leaky integrator has a larger time constant for its integration and leakage, integrating incoming spikes generated in the axon hillock in the timescale from tens of seconds to minutes, due to its slow rate of leakage $f$, as described in Equation (3). When the potential in the axonal leaky integrator exceeds $w_{p}$, which denotes the high threshold (rising edge), the persistent firing will be triggered, and the firing pattern is determined by the parameter set of $(a, b, c, d, e)_{p}$. If there are no somatic spikes accumulated in the axon, the potential \$w\$ decreases at the rate of $f$. When $w$ reaches the low threshold (falling edge), the axon $w_{n}$ returns to passive conduction mode, and the $a, b, c, d, e$ variables in the model are reset to $(a, b, c, d, e)_{n}$.

We have simulated the persistent firing neuronal behaviors under two stimulation protocols, with the parameters summarized in Table 1. The simulation results under step/pause stimulation protocol [12] are shown in Figure 1. We applied 1-second current step of $500 \mathrm{pA}$ during each 10-second sweep to the simulation model. During the sweeps of the step/pause input stimulus, it is observed that the somatically evoked action potentials appear when the synaptic input stimulus is presented and disappear when the stimulus is removed (Figure 1(a)). When $w$ accumulates to a level higher than the high threshold $w_{p}$, persistent firing occurs (Figure 1(a)), 12th sweep), after which $w$ decreases monotonically due to axonal leakage and no stimulus (Figure 1(d)). The persistent firing ends when $w$ is lower than the low threshold $w_{n}$. It should be noted that in this model, the resting potential of persistent firing spikes is about $20 \mathrm{mV}$ below the one of somatically evoked action potentials, which fits well with the data from current-clamp recordings in the persistent firing neurons [12] [14]. We emulate this phenomenon through switching the parameter $c$ in Equation (4) between $c_{p}$ and $c_{n}$.

The variation of persistent firing frequency was also simulated (Figure 1(b) and Figure 1(c)). As the firing frequency can be tuned by the parameter $b$ in Equation (2), the decrease of persistent firing frequency observed in the current-clamp experiment can be emulated in the model through defining the following relationship between parameter $b$ and $f$.

Table 1. Simulation parameters.

\begin{tabular}{cc}
\hline Step/Pause protocol & Long Pulse protocol \\
\hline$(a, b, c, d, e)_{p}=(0.1,5,-85,0,0)$ & $(a, b, c, d, e)_{p}=(0.1,0.3,-85,0,0)$ \\
$(a, b, c, d, e)_{n}=(0.1,0.2,-65,2,0.001)$ & $(a, b, c, d, e)_{n}=(0.1,0.2,-65,2,0.012)$ \\
$f=5 \times 10^{-4}$ & $f=8 \times 10^{-4}$ \\
$w_{n}=0.2, w_{p}=0.84$ & $w_{n}=0.2, w_{p}=2.1$ \\
$I=500 \mathrm{pA}$ & $I=15 \mathrm{pA}$ \\
\hline
\end{tabular}



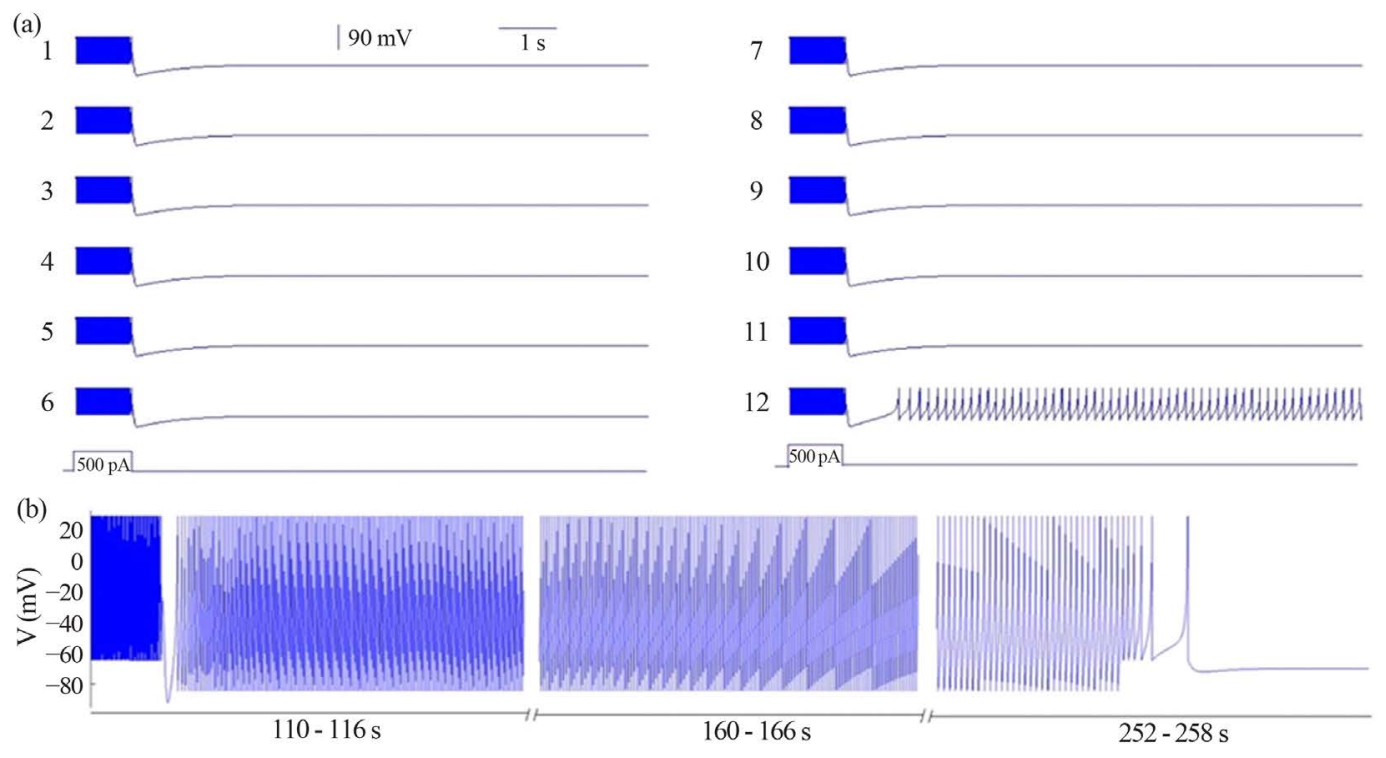

(c)

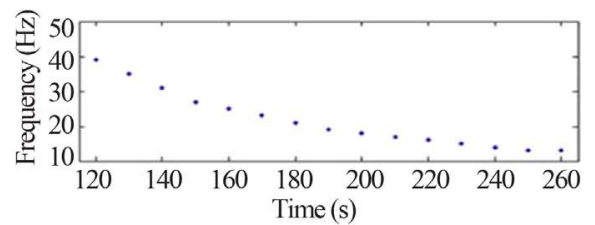

(d)

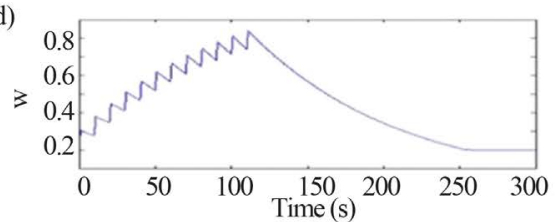

\begin{abstract}
Figure 1. Simulation of persistent firing neuron model. (a) The waveform of membrane potential. The simulation used the same stimulation protocol as the experiment of whole-cell current-clamp recording by Sheffield et al [12]. To evoke persistent firing, 1-s current step of $500 \mathrm{pA}$ was delivered to the neuron during each 10-s sweep (left, sweeps 1 - 6; right, sweeps 7 - 12). In this simulation, persistent firing was evoked after the 12th sweep, and the total number of evoked action potentials before persistent firing was 1278. (b) The waveform of persistent firing, which lasted over 2 minutes. The total number of evoked axonal action potentials was 3073. The simulation reflects the decrease of instantaneous firing frequency, which was shown in the current-clamp experiment. (c) The instantaneous firing frequency of persistent firing action potentials, which decreased from $40 \mathrm{~Hz}$ at the onset of persistent firing to $12 \mathrm{~Hz}$ at the end of persistent firing as the parameter $b$ decreased in this simulation. (d) The time response of $w$, which represents the hypertheoretical potential of the axonal leaky integrator.
\end{abstract}

if persistent firing action potential is generated, then $b \leftarrow b(1-f)$

The instantaneous firing frequency of axon-evoked action potentials attains its maximum $(40 \mathrm{~Hz})$ at the onset of persistent firing, and decreases over time till the end of persistent firing.

We also applied the stimulation with a 40-second long pulse of $15 \mathrm{pA}$ current to the persistent firing neuron model described by Equations (1)-(7) with a different parameter set (Table 1). The simulation results are shown in Figure 2. The repeated somatic current stimuli eventually trigger the persistent firing that outlasts the current stimuli by more than 30 seconds.

By tuning the parameters of $e, f, w_{p}$ and $w_{n}$, we can set different time scales for the axonal slow integration, allowing the model to accommodate different types of neurons with different persistent firing behaviors.

\title{
3. Model Analysis
}

In this section, we present the mathematical analysis on the conditions which guarantee the existence of the persistent firing behavior in the neuron. Let $X=[u v w]^{\prime}$. Equations (1)-(3) can be rewritten as:

$$
X^{\prime}=F(X)+H I=X^{\mathrm{T}} A X+B X+C+H I
$$

where $I$ is the input current, $A, B, C$ and $H$ are the corresponding matrices determined by 

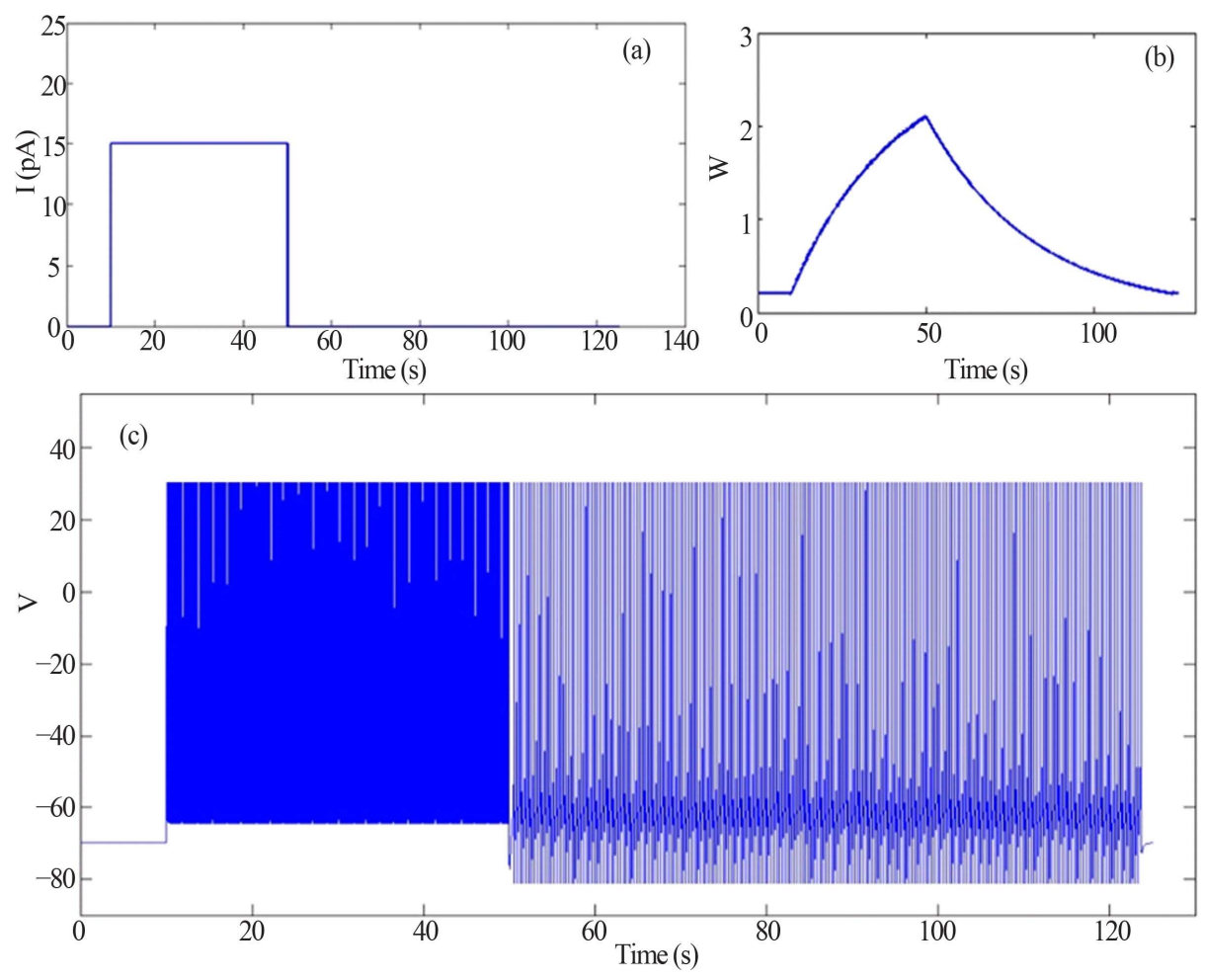

Figure 2. Simulation of persistent firing neuronal behaviors with long pulse stimulation protocol. (a) The input stimulus. A pulse of current with $15 \mathrm{pA}$ amplitude lasts 40 seconds as the synaptic input to the neuron. (b) Time response of $w$, the variable describing the potential of the axonal leaky integrator. (c) Time response of $v$, which describes the membrane or axon potential. The first part is the fast spiking waveform of $v$ when the 15pA current step is present, and the second part is the persistent firing waveform of $v$.

Equations (1)-(3). When the input current $I$ is not present, the equilibrium points of the dynamic system in Equation (8) are the solutions of the Equation $F(X)=0$, the two equilibrium points are obtained $X_{1}=[-12.5(5-b+\sqrt{\Delta})-12.5(5-b+\sqrt{\Delta}) 0]^{\prime}$ and $X_{2}=[-12.5(5-b-\sqrt{\Delta})-12.5(5-b-\sqrt{\Delta}) 0]^{\prime}$.

Here, we assume that $\Delta=2.6+b^{2}-10 b>0$ and it is noted that $X_{1}$ is a stable equilibrium point while $X_{2}$ is unstable. Based on the analysis of stability of the equilibrium points and the nonlinear dynamic characteristic of Equation (8), the following conditions can be summarized to guarantee the existence of the persistent firing behavior.

Condition 1. The input current $I$ satisfies that $\forall v \in R$ such that $0.04 v^{2}+(5-b) v+140 \geq 0$, i.e, $I \geq\left|\min \left\{0.04 v^{2}+(5-b) v+140\right\}\right|$.

Condition 2. The condition $w>w_{p}$ is satisfied no later than the time when $I$ is removed.

Condition 3. When $w>w_{p}$, the parameter $c$ is denoted as $c_{p}$ in Equation (5) (during the persistent firing period) is chosen to be the value such that $c>-12.5(5-b-\sqrt{\Delta})$.

Condition 1 means that there is no equilibrium point for the equation $F(X)+H I=0$ which gives $X^{\prime} A X+B X+C+H I=0$. Otherwise, $X$ may approach the equilibrium point and not change any more even when $I$ is present. In this case, it is impossible for persistent firing to exist. Condition 2 requires that the neuron fires a sufficient number of spikes to ensure that $w>w_{p}$ before $I$ is removed. Condition 3 implies that, during the persistent firing period, $0.04 v^{2}+(5-b) v+140>0$ should be guaranteed at the interval $-12.5(5-b-\sqrt{\Delta}) \leq v \leq c_{p}$.

In the above analysis, we choose $b$ to be such that $\Delta>0$. Now we analyze the case that $\Delta \leq 0$. It can be 
shown that there is no real equilibrium point or at most a unstable equilibrium point for the equation $F(X)=0$. Then Condition 1 is achieved even if $I=0$. The persistence firing can be always observed no matter whether the Conditions 2 and $\mathbf{3}$ are satisfied or not. However, in this case, the nonlinear system $X=F(X)+H I$ is unstable. This is the reason that generally $b$ is suggested to be chosen as a value such that $\Delta>0$.

The geometrical approach provides a clear and insightful perspective of investigating the characteristics of dynamical system of neuron [15], and is therefore adopted to analyze the persistent firing model. We choose conveniently to conduct geometrical analysis on the phenomenon of persistent firing due to a long-lasting pulse stimulation (Figure 2 and Figure 3(a)) rather than on that due to a pulse train (Figure 1), since the geometry of the former is less complicated yet retains significant information regarding the time evolution of the dynamical
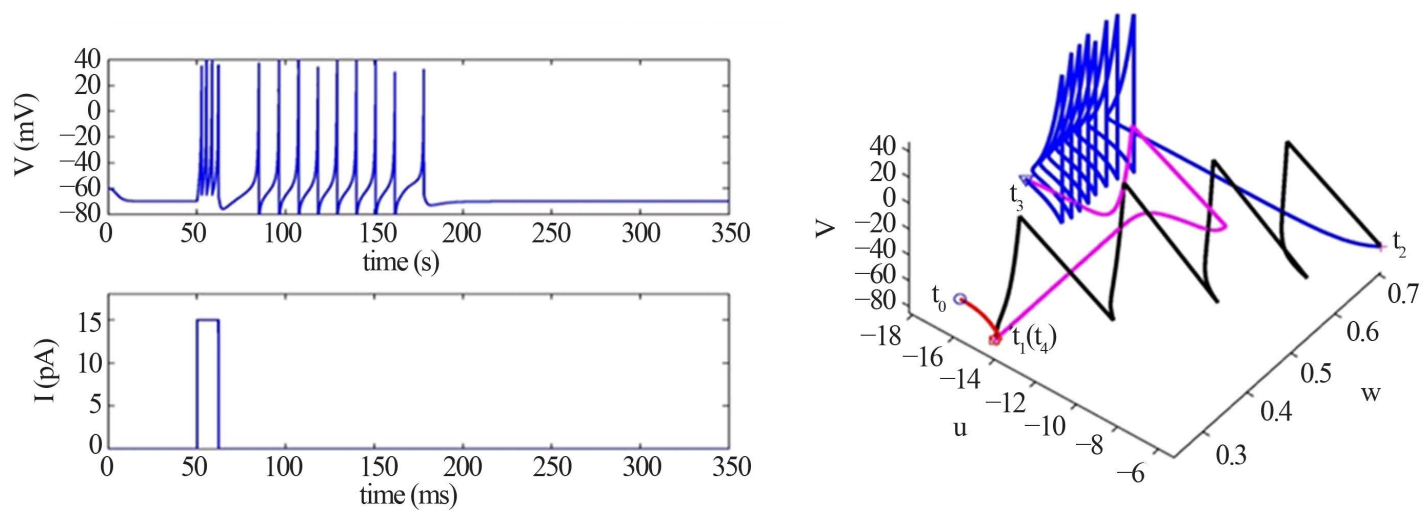

(a)

(b)

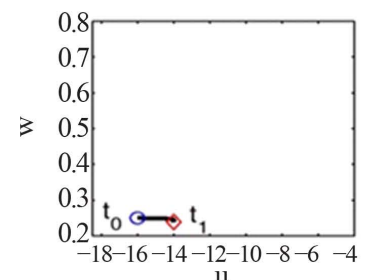

(al)

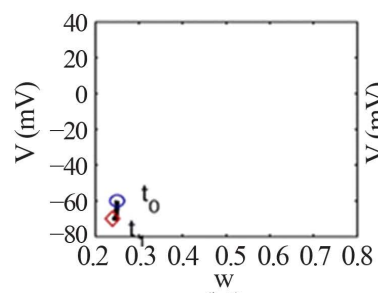

(b1)

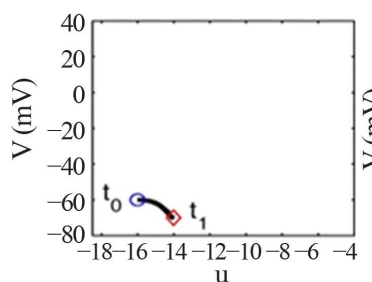

(c1)

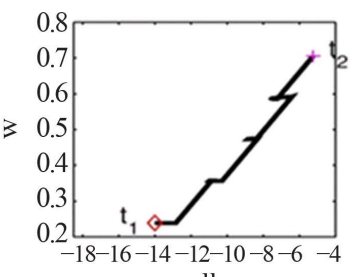

(a2)

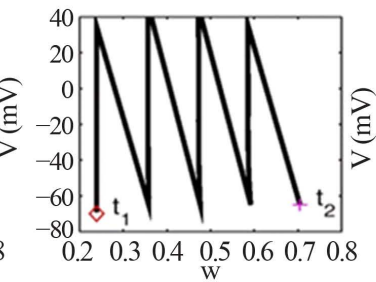

(b2)

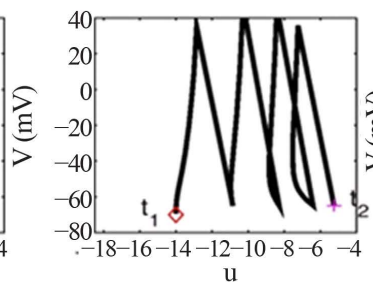

(c2)

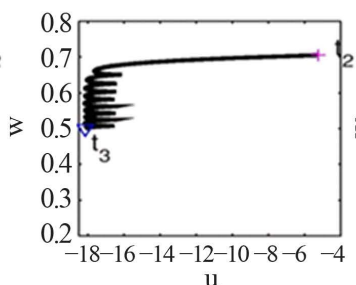

(a3)

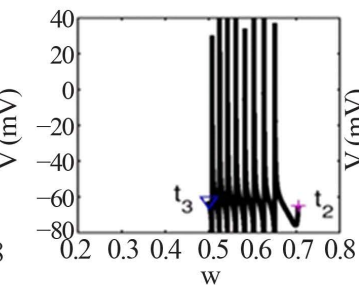

(b3)

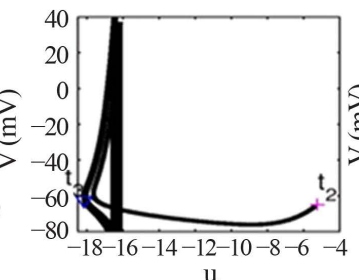

(c3)

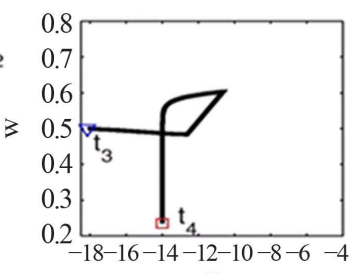

$\mathrm{u}$
(a4)

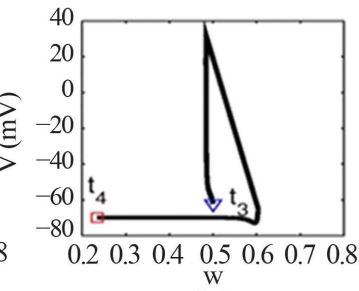

(b4)

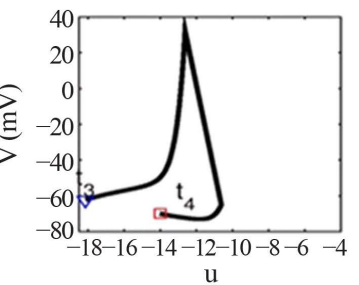

(c4)

(c)

Figure 3. (a) Persistent firing induced by an input current pulse; (b) Trajectory of state variables $u, v, w$ in three-dimensional phase space; (c) The three rows are projections of the 3-D trajectory. Figure 3(b) of persistent firing on the $u w$ (a1-a4), wv (b1-b4), and $u v$ (c1-c4) phase planes, respectively; the four columns represent the four stages of the whole process of persistent firing: stage 1 (a1, b1, c1), stage 2 (a2, b2, c2), stage 3 (a3, b3, c3), and stage 4 (a4, b4, c4), respectively. 
system of neuron, such as the stable equilibrium and the threshold values, as shown in Figure 3(b) where the geometrical trajectory of the state variables $u, v, w$ is plotted in three-dimensional phase space. It is worth mentioning that a new set of values in Table 2 is assigned to the model's parameters to further reduce the complexity of the geometry, as shown in Figure 3(a) and Figure 3(b). For the convenience of analysis, the projections of the 3-D trajectory on the $u w, w v$, and $u v$ phase planes are illustrated in Figure 3(c), where five points in time ( $t \_0, t \_1, t \_2, t \_3, t \_4$ ) are labeled on the curves, so as to partition the whole course of persistent firing into four temporal stages:

Stage 1: During the time range of $t_{0} \rightarrow t_{1}$, the system begins with its initial state at $t_{0}$, converges to a stable equilibrium, stay there until $t_{1}$. The stable equilibrium can be calculated to be $[-70,-14,0]^{\prime}$.

Stage 2: During the time range of $t_{1} \rightarrow t_{2}$, the system exhibits action potential evoked by a rectangular current signal, which also leads to the persistent firing in the next stage.

Stage 3: During the time range of $t_{2} \rightarrow t_{3}$, the system exhibits persistent firing, which is triggered at $t_{2}$ when $w$ reaches the upper threshold $w_{p}=0.7$, and terminates at $t_{3}$ when $w$ reaches the lower threshold value $w_{n}=0.5$.

Stage 4: During the time range of $t_{3} \rightarrow t_{4}$, the system returns to the state of stable equilibrium and rests there.

\section{Neuromorphic Model of the Artificial Neuron}

Since the last two decades, there has been a continuing interest in developing the neuromorphic circuits and systems that mimic the operation of biological neurons and brains, which enables considerably faster and more energy-efficient emulations of the neurons and neural systems. Due to the computational simplicity of our model, it is rather straightforward to implement the proposed neuron model in hardware, either in digital circuits or analog circuits. There have been several circuit implementations of Izhikevich neuron model and its variants [16] [17]. Thus in our case it is intuitive to add a leaky integrator emulating the axon, as well as the switching devices for selecting one of two sets of $a, b, c, d, e$ parameters, which may be stored in memory devices, e.g. nonvolatile memory.

A conceptual design of the proposed artificial neuron are shown in Figure 4, where the axonal spikes are originated from the unstable state of the neuron circuit and the artificial neuron is considered as a parameter-dependent dynamic system. The parameters of such dynamic system can be stored in non-volatile memory devices. Through modifying the parameter values in the memory, the spiking neuron unit can alter the axonal dynamics between the passive conduction mode and the persistent firing mode.

We have built the neuromorphic model based on the concept design with SPICE and Verilog-A languages, and simulated the model in Synopsys ${ }^{\circledR}$ HSPICE Simulator. The circuit model is shown in Figure 5(a) and Figure 5(b). The soma membrane circuit (Figure 5(a)) essentially implements the Izhikevich neuron model [10], which includes a SPICE block describing Equations (1)-(2), an adder, a Schmitt trigger and two NMOS transistors (M1, M2) as switches. The stimulus $I$ is the only external input to the soma unit, which also requires to fetch a parameter set of $a, b, V_{p}$ and $c$ from its memory during the operation. The Schmitt trigger implemented in Verilog-A, compares the membrane potential $v$ with $V_{p}$ (peak of the spike, typically $30 \mathrm{mV}$ ) and $c$ (rest potential, typically $-65 \mathrm{mV}$ ), and produces the output $C_{v}$, of VDD only when $>V_{p}$, and GND only when $v<c$. When $c_{v}$ is high (VDD), which means a spike is generated, the two NMOS switches (M1, M2) are turned on thus the potentials of $u$ and $v$ are reset to $u_{\text {new }}$ and $c$, respectively. When $C_{v}$ is low (GND), the potentials of \$u $\$$ and $v$ remain to be solely determined by Equations (1)-(2) in the SPICE block.

\begin{tabular}{ccc} 
Table 2. Simulation parameters. & \\
\hline$(a, b, c, d, e)_{p}$ & $=$ & $(0.1,0.3,-85,0,0)$ \\
$(a, b, c, d, e)_{n}$ & $=$ & $(0.1,0.2,-85,2,0.12)$ \\
$f$ & $=$ & $5 \times 10^{-4}$ \\
$w_{n}$ & $=$ & 0.5 \\
$w_{p}$ & $=$ & 0.7 \\
$I$ & $=$ & $15 \mathrm{pA}$ \\
\hline
\end{tabular}




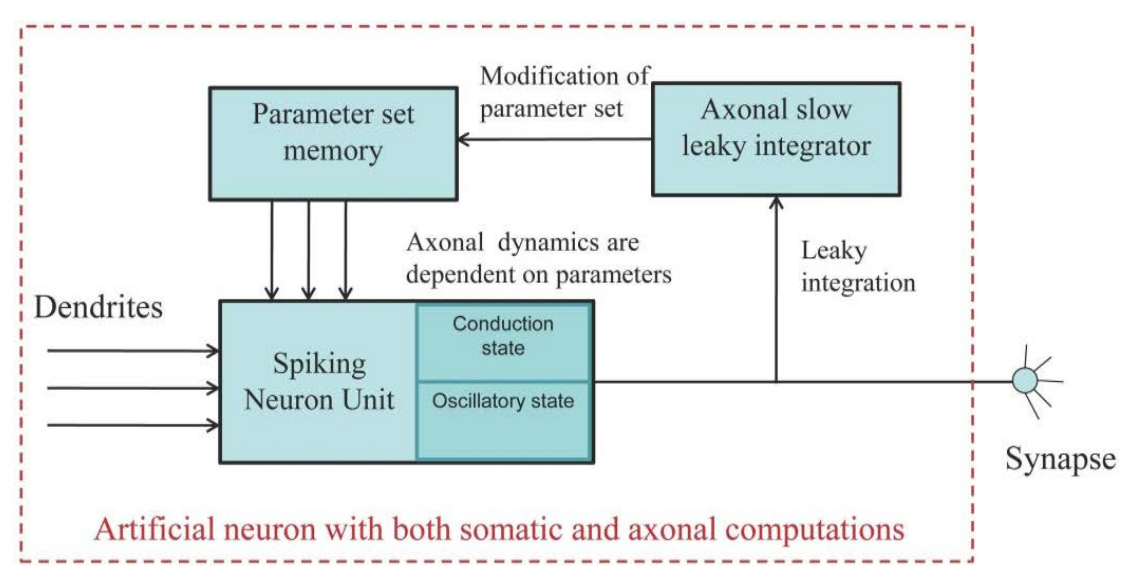

Figure 4. Conceptual design of the proposed artificial neuron.

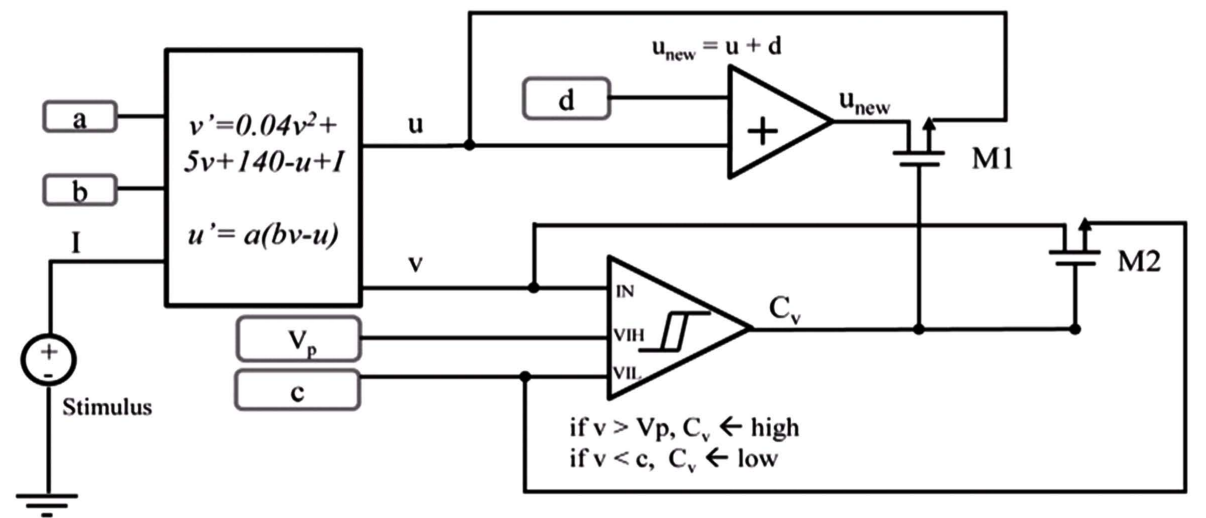

(a)
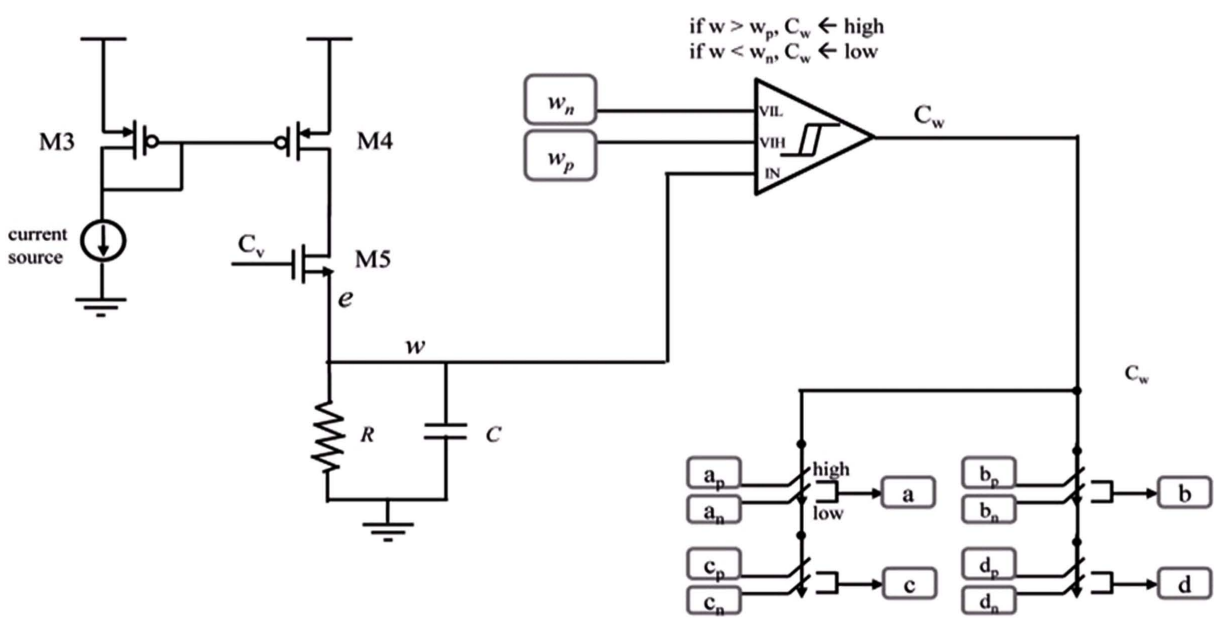

(b)

Figure 5. (a) The soma membrane circuit; (b) The axon circuit.

In the axon circuit (Figure 5(b)), a parallel RC circuit implements the axonal leaky integrator. When the soma membrane is generating a spike, $C_{v}$ is high (VDD) and NMOS M5 will be turned on and pull the current from the current mirror (M3, M4), and charge the RC leaky integrator, increasing the axon potential $w$. The potential $w$ is compared with $w_{n}$ and $w_{p}$ by a Schmitt trigger, which produces the output $C_{w}$ of VDD when $w>w_{p}$ (the persistent firing should start) and GND when $w<w_{n}$ (the persistent firing should stop). The $C_{w}$ signal controls five 2-way switches, which subsequently determine the value of $x$ to be $x_{n}$ or $x_{p}$, where 
$x \in\{a, b, c, d, e\}$, and the neuron spiking dynamics.

Based on the long-pulse stimulation protocol and the circuit parameters given in Table 3, the simulation results of the circuit are shown in Figure 6. It should be noted that the values of the stimulus current $I$ and simulation time here are different from those discussed in the previous section, with the purpose of achieving simpler implementation in the circuits. Nevertheless, it can be clearly seen that after we continuously applied the stimulus current for a period of time and stopped the input, the persistent firing of spikes was triggered, lasted for a similar duration, and finally stopped, depending on the level of axonal integrator potential $w$.

\section{Persistent Firing Spiking Network}

We have used this model to simulate a spiking network of 1000 randomly connected neurons. The network takes into account of axonal conduction delays [18] and spike-timing-dependent plasticity (STDP), which is considered to be "polychromous" [13]. The network consists of 800 excitatory neurons with regular spiking pattern [11] and 200 inhibitory neurons. As around 80\% of EGFP-positive interneurons were found to have persistent firing behavior by repeated somatic current injection [12], we set in the $80 \%$ simulation, or 160 of the inhibitory neurons to be capable of persistent firing while the remaining $20 \%$ are fast spiking inhibitory neurons.

Each excitatory neuron is connected to 100 random neurons, and each inhibitory neuron, including persistent firing neuron, is randomly connected to 100 excitatory neurons only. Each synaptic connection has a fixed integer conduction delay between $1 \mathrm{~ms}$ and $20 \mathrm{~ms}$. The conduction delay in the range of $1-20 \mathrm{~ms}$ is randomly as-

Table 3. Circuit simulation parameters.

\begin{tabular}{ccc}
\hline$(a, b, c, d, e)_{p}$ & $=$ & $(0.1 \mathrm{~V}, 0.3 \mathrm{~V},-85 \mathrm{mV}, 0 \mathrm{~V}, 0.1 \mathrm{~mA})$ \\
$(a, b, c, d, e)_{n}$ & $=$ & $(0.1 \mathrm{~V}, 0.2 \mathrm{~V},-65 \mathrm{mV}, 2 \mathrm{mV}, 0.1 \mathrm{~mA})$ \\
$f$ & $=$ & 0.2 \\
$w_{n}$ & $=$ & $40 \mathrm{mV}$ \\
$w_{p}$ & $=$ & $90 \mathrm{~mA}$ \\
\hline
\end{tabular}

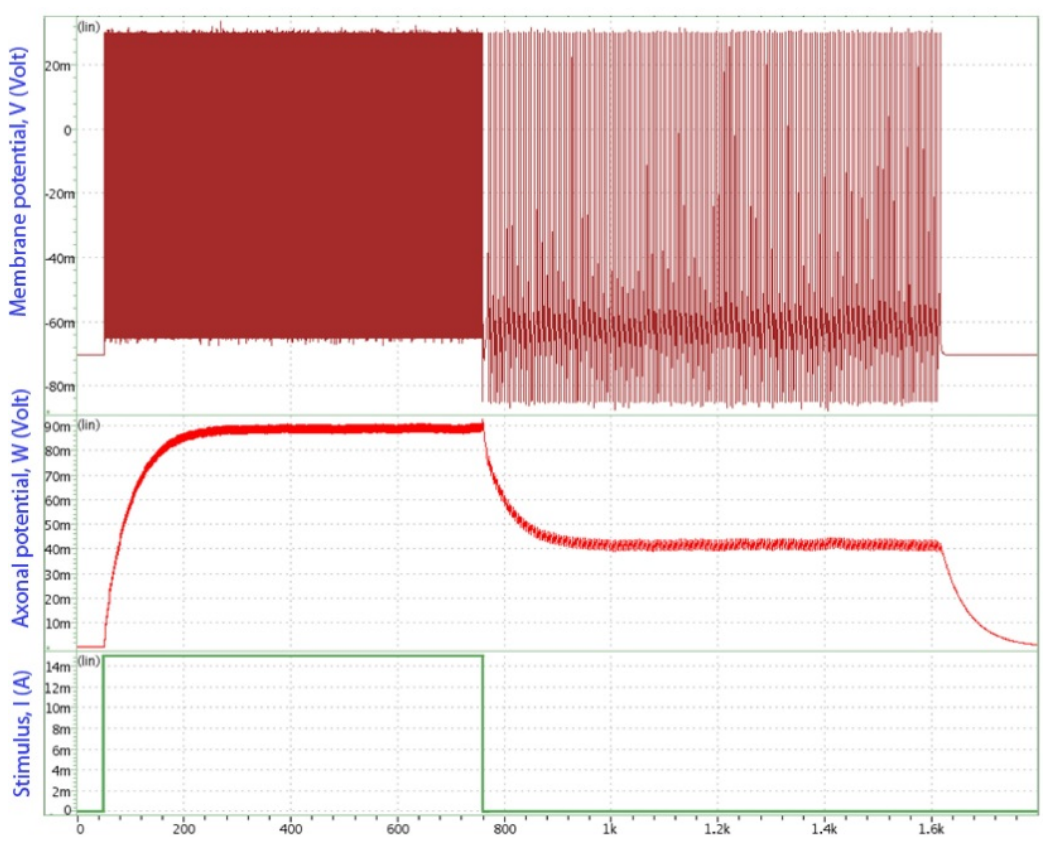

Figure 6. Circuit simulation results of the artificial neuron, showing that after a continuous applied stimulus stops, the slow integration in the axon leads to persistent firing of spikes. Top: the membrane potential $v$; Middle : the potential of axonal integrator $w$; Bottom: the stimulus current $I$. 
signed to all excitatory synapses, while the $1 \mathrm{~ms}$ delay are assigned to all inhibitory synapses [13]. The synaptic connections are modified according to the STDP rule [19]. The implementation of the STDP function is based on Equation (9):

$$
W(\Delta t)= \begin{cases}A^{+} \mathrm{e}^{-\Delta t / \tau^{+}}, & \text {for } \Delta t>0 \\ -A^{-} \mathrm{e}^{\Delta t / \tau^{-}} & \text {for } \Delta t<0\end{cases}
$$

where $\Delta t=t_{\text {post }}-t_{\text {pre }}, W(\Delta t)$ represents the strength of synapse connection, $A^{+}$and $A^{-}$depend on the maximum synaptic strength, and $\tau^{+}$and $\tau^{-}$are time constants determining the time window of STDP.

A simplified example of the spiking network structure is illustrated in Figure 7(a). The raster plots of 1-second firing activities in the 1000-neuron spiking network with persistent firing inhibitory interneurons are shown in Figure 7(b). One can see from Figure 7(b) that the network exhibits asynchronous dynamics. Different rhythmic activities can be identified, ranging from $3 \mathrm{~Hz}$ to $8 \mathrm{~Hz}$. Dense vertical columns indicate there are occasional episodes of synchronized firings. As the firing rate of the inhibitory neurons (fast spiking) is higher than that of excitatory neurons (regular spiking), there are generally more firings for inhibitory neurons. The

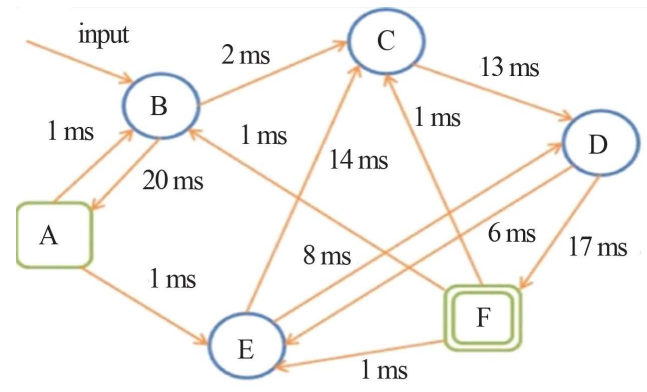

Excitatory Neuron

(a)
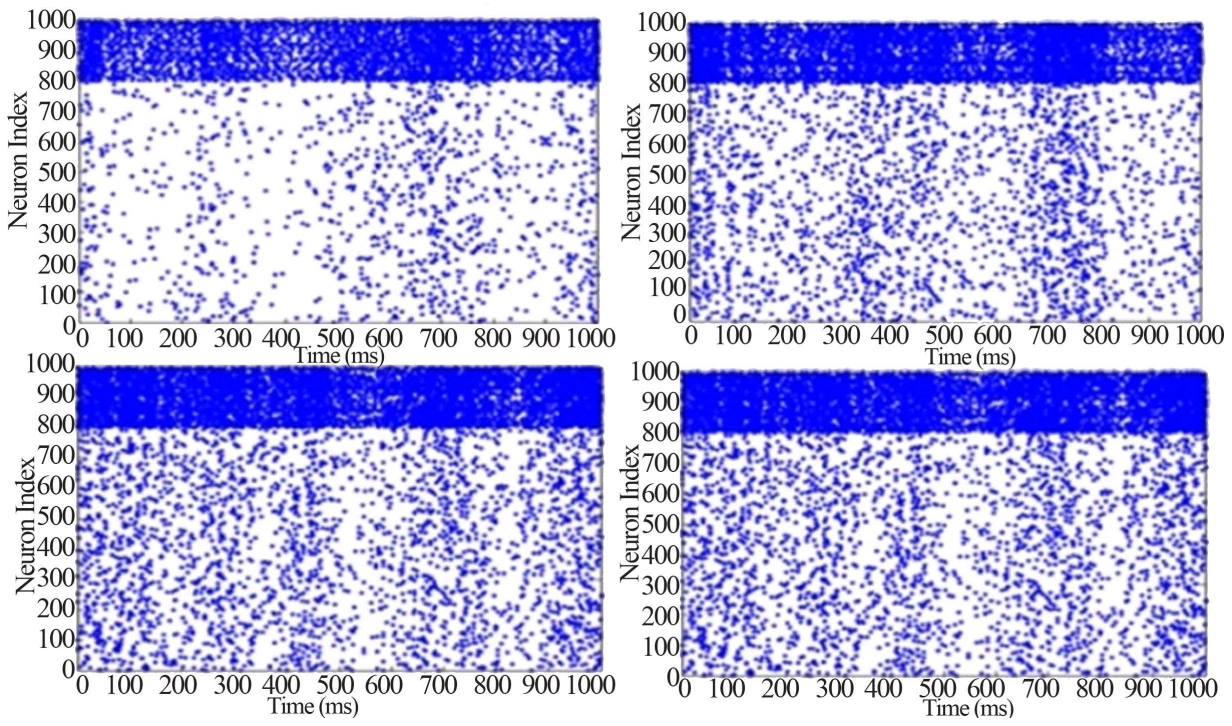

(b)

Figure 7. (a) A simplified example of the persistent firing spiking network structure. Among the neurons ranging from $\mathrm{A}$ to $\mathrm{F}$ in the network, $(\mathrm{B}, \mathrm{C}, \mathrm{D}, \mathrm{E})$ represent the excitatory neurons, and (A, F) represent inhibitory neurons in which $\mathrm{F}$ is capable for persistent firing. (b) Raster plots of spike activities in the spiking network of 1000 neurons including persistent firing inhibitory interneurons. Each raster plot shows the 1-second segment of the firing activity. Neurons indexed from 1 to 800 are excitatory neurons, and neurons indexed from 801 to 1000 are inhibitory. Within the 200 inhibitory neurons, there are 160 randomly selected neurons with persistent firing capability. Horizontally continuous dotted lines with neuron index in the range of 801 - 1000 indicate there are persistent firings of action potentials in inhibitory neurons. 
persisting firing of inhibitory interneurons in the network, leads to reciprocal inhibition in a longer timescale and thus shut down the activities for a longer period (Figure 7(b) middle column). The synchronization of persistent firing interneurons could contribute to the beta and gamma oscillation, whose frequency ranges are close to the persistent firing frequency [20].

\section{Discussion}

The possible functions of the persistent firing were suggested to be related to working memory [12]. The ability to maintain the persistent firing of action potentials without on-going stimulation provides a mechanism of storing the information for a short period of time. This mechanism is similar to our working memory [21]-[23], which actively holds a limited amount of information [24] in the absence of stimuli. Working memory has been extensively explored from perspectives of highly abstract top levels in the domains of psychology, neuroscience and anatomy, but there are much less work from perspectives of bottom level of biological neurons [25]-[30]. It is still unknown how working memory is represented within a population of cortical neurons.

The presented simple model of persistent firing neurons enables further investigation of possible functions of persistent firing, especially the relationship between working memory and persistent firing, and the neural correlate of working memory. Recently, we have proposed a model of short term persistent habituation [31], consisting of a persistent firing neuron and a habituating synapse, to explore the presynaptic learning and memory. The interaction of persistent firing axonal and presynaptic processes increases the retention time of the synaptic conductance and therefore the recovery time, and continues the learning of short term habituation for the duration of persistent firing. This leads to a working memory for habituation.

Through incorporating the persistent firing dynamics in spiking networks with axonal delays and STDP learning rules, we can further investigate the interaction of polychronization and persistent neural activities. In the polychronous spiking network, the number of co-existing polychronous groups far exceeds the number of neurons in the network, resulting in an unprecedented memory capacity of the system [13]. Thus it would be interesting to investigate how working memory is presented in the polychronous network, and simulate a bioplausible working memory system with increased memory capacity. To this end, we are working towards the development of artificial cognitive memory with the objective of developing a novel function-driven memory technology in comparison to conventional density-driven storage technology [32]. The models of persistent firing neuron and spiking network presented in this paper can be used in the simulation of cognitive memory architectures.

Due to the computational simplicity of our model, it is straightforward to implement the model in hardware. We have developed a neuromorphic model of persistent firing neurons [33], which reproduces the neuronal persistent firing behavior by integrating somatic and axonal computational processes of different timescales. Considering there are many existing VLSI implementations of Izhikevich neuron model and its variants [16] [34][36], the proposed neuron model can be conveniently implemented in silicon by incorporating the axonal leaky integrator into the Izhikevich VLSI designs, enabling a considerably faster emulation of the neural systems with persistent neural activity in a highly parallel manner.

\section{Conclusion}

Based on the recent discovery in neurophysiology which revealed that interneurons can slowly integrate spiking, share the output across a coupled network of axons and respond with persistent firing even in the absence of input to the soma or dendrites, we proposed a new model of persistent firing neuron to bridge the gap between the conventional models and the newly-discovered phenomenon. In this work, we presented and discussed the mathematical and neuromorphic models of the artificial neuron, as well as a persistent firing polychronous spiking network which exhibits asynchronous dynamics. The artificial neuron we proposed, being computationally efficient yet bio-plausible, would be useful to construct and simulate the large scale models of animal or human cortex, which provides a neuromorphic platform for further investigation of the possible functions of persistent firing and their roles in animal and human brain, especially for exploring the relationship between working memory and persistent firing spiking network-based memory and the bio-inspired computer systems.

\section{References}

[1] Lapicque, L. (1907) Recherches quantitatives sur l'excitation electrique des nerfs traitee comme une polarization. Jour- 
nal of Physiol Pathol Générale, 9, 620-635.

[2] Sandberg, A. and Bostrom, N. (2008) Whole Brain Emulation: A Roadmap. Future of Humanity Institute, Oxford University, Technical Report \#2008-3.

[3] Hodgkin, A.L. and Huxley, A.F. (1952) A Quantitative Description of Membrane Current and Its Application to Conduction and Excitation in Nerve. The Journal of Physiology, 117, 500-544. http://dx.doi.org/10.1113/jphysiol.1952.sp004764

[4] Brette, R., Rudolph, M., Carnevale, T., Hines, M., Beeman, D., Bower, J.M., Diesmann, M., Morrison, A., Goodman, P.H., Harris Jr., F.C, et al. (2007) Simulation of Networks of Spiking Neurons: A Review of Tools and Strategies. Journal of Computational Neuroscience, 23, 349-398. http://dx.doi.org/10.1007/s10827-007-0038-6

[5] Mihalas, S. and Niebur, E. (2009) A Generalized Linear Integrate-and-Fire Neural Model Produces Diverse Spiking Behaviors. Neural Computation, 21, 704-718. http://dx.doi.org/10.1162/neco.2008.12-07-680

[6] Fitzhugh, R. (1961) Impulses and Physiological States in Theoretical Models of Nerve Membrane. Biophysical Journal, 1, 445-466. http://dx.doi.org/10.1016/S0006-3495(61)86902-6

[7] Morris, C. and Lecar, H. (1981) Voltage Oscillations in the Barnacle Giant Muscle Fiber. Biophysical Journal, 35, 193-213. http://dx.doi.org/10.1016/S0006-3495(81)84782-0

[8] Rose, R.M. and Hindmarsh, J.L. (1989) The Assembly of Ionic Currents in a Thalamic Neuron I. The Three-Dimensional Model. Proceedings of the Royal Society of London. Series B. Biological Sciences, 237, 267-288. http://dx.doi.org/10.1098/rspb.1989.0049

[9] Wilson, H.R. (1999) Simplified Dynamics of Human and Mammalian Neocortical Neurons. Journal of Theoretical Biology, 200, 375-388. http://dx.doi.org/10.1006/jtbi.1999.1002

[10] Izhikevich, E.M. (2001) Resonate-and-Fire Neurons. Neural Networks, 14, 883-894. http://dx.doi.org/10.1016/S0893-6080(01)00078-8

[11] Izhikevich, E.M. (2003) Simple Model of Spiking Neurons. IEEE Transactions on Neural Networks, 14, 1569-1572. http://dx.doi.org/10.1109/TNN.2003.820440

[12] Sheffield, M.E.J., Best, T.K., Mensh, B.D., Kath, W.L. and Spruston, N. (2011) Slow Integration Leads to Persistent Action Potential Firing in Distal Axons of Coupled Interneurons. Nature Neuroscience, 14, 200-207. http://dx.doi.org/10.1038/nn.2728

[13] Izhikevich, E.M. (2006) Polychronization: Computation with Spikes. Neural Computation, 18, 245-282. http://dx.doi.org/10.1162/089976606775093882

[14] Ning, N., Yi, K.J., Huang, K.J. and Shi, L.P. (2011) Axonal Slow Integration Induced Persistent Firing Neuron Model. Lecture Notes in Computer Science, 7062, 469-476. http://dx.doi.org/10.1007/978-3-642-24955-6 56

[15] Izhikevich, E.M. (2007) Dynamical Systems in Neuroscience: The Geometry of Excitability and Bursting. The MIT Press, Cambridge.

[16] Wijekoon, J.H. and Dudek, P. (2008) Compact Silicon Neuron Circuit with Spiking and Bursting Behaviour. Neural Networks, 21, 524-534. http://dx.doi.org/10.1016/j.neunet.2007.12.037

[17] Wijekoon, J.H. and Dudek, P. (2008) Integrated Circuit Implementation of a Cortical Neuron. Proceedings of the IEEE International Symposium on Circuits and Systems, Seattle, 18-21 May 2008, 1784-1787.

[18] Swadlow, H.A. (1985) Physiological Properties of Individual Cerebral Axons Studied in Vivo for as Long as One Year. Journal of Neurophysiology, 54, 1346-1362.

[19] Song, S., Miller, K.D. and Abbott, L.F. (2000) Competitive Hebbian Learning through Spike-Timing-Dependent Synaptic Plasticity. Nature Neuroscience, 3, 919-926. http://dx.doi.org/10.1038/78829

[20] Bartos, M., Vida, I. and Jonas, P. (2007) Synaptic Mechanisms of Synchronized Gamma Oscillations in Inhibitory Interneuron Networks. Nature Reviews Neuroscience, 8, 45-56. http://dx.doi.org/10.1038/nrn2044

[21] Baddeley, A. (1992) Working Memory. Science, 255, 556-559. http://dx.doi.org/10.1126/science.1736359

[22] Durstewitz, D., Seamans, J.K. and Sejnowski, T.J. (2000) Neurocomputational Models of Working Memory. Nature Neuroscience, 3, 1184-1191. http://dx.doi.org/10.1038/81460

[23] Egorov, A.V., Hamam, B.N., Fransen, E., Hasselmo, M.E. and Alonso, A.A. (2002) Graded Persistent Activity in Entorhinal Cortex Neurons. Nature, 420, 173-178. http://dx.doi.org/10.1038/nature01171

[24] Miller, G.A. (1956) The Magical Number Seven, Plus or Minus Two: Some Limits on Our Capacity for Processing Information. Psychological Review, 63, 81-97. http://dx.doi.org/10.1037/h0043158

[25] D’Esposito, M., Detre, J.A., Alsop, D.C., Shin, R.K., Atlas, S. and Grossman, M. (1995) The Neural Basis of the Central Executive System of Working Memory. Nature, 378, 279-281. http://dx.doi.org/10.1038/378279a0

[26] Goldman-Rakic, P.S. (1995) Cellular Basis of Working Memory. Neuron, 14, 477-485. 
http://dx.doi.org/10.1016/0896-6273(95)90304-6

[27] Miller, E.K., Erickson, C.A. and Desimone, R. (1996) Neural Mechanisms of Visual Working Memory in Prefrontal Cortex of the Macaque. The Journal of Neuroscience, 16, 5154-5167.

[28] Amit, D. and Mongillo, G. (2003) Spike-Driven Synaptic Dynamics Generating Working Memory States. Neural Computation, 15, 565-596. http://dx.doi.org/10.1162/089976603321192086

[29] O’Reilly, R.C. and Frank, M.J. (2006) Making Working Memory Work: A Computational Model of Learning in the Prefrontal Cortex and Basal Ganglia. Neural Computation, 18, 283-328. http://dx.doi.org/10.1162/089976606775093909

[30] Szatmary, B. and Izhikevich, E.M. (2010) Spike-Timing Theory of Working Memory. PLoS Computational Biology, 6, e1000879. http://dx.doi.org/10.1371/journal.pcbi.1000879

[31] Ramanathan, K., Ning, N., Dhanasekar, D., Li, G., Shi, L.P. and Vadakkepat, P. (2012) Presynaptic Learning and Memory with a Persistent Firing Neuron and a Habituating Synapse: A Model of Short Term Persistent Habituation. International Journal of Neural Systems, 22, Article ID: 1250015. http://dx.doi.org/10.1142/S0129065712500153

[32] Shi, L.P., Yi, K.J., Ramanathan, K., Zhao, R., Ning, N., Ding, D. and Chong, T.C. (2011) Artificial Cognitive Memory-Changing from Density Driven to Functionality Driven. Applied Physics A, 102, 865-875. http://dx.doi.org/10.1007/s00339-011-6297-0

[33] Ning, N., Huang, K.J. and Shi, L.P. (2012) Artificial Neuron with Somatic and Axonal Computation Units: Mathematical and Neuromorphic Models of Persistent Firing Neurons. Proceedings of the 2012 International Joint Conference on Neural Networks (IJCNN), Brisbane, 10-15 June 2012, 1-7.

[34] van Schaik, A., Jin, C., McEwan, A. and Hamilton, T.J. (2010) A Log-Domain Implementation of the Izhikevich Neuron Model. Proceedings of 2010 IEEE International Symposium on Circuits and Systems (ISCAS), Paris, 30 May-2 June 2010, 4253-4256.

[35] Li, G.Q., Ning, N., Ramanathan, K., Wei, H., Pan, L. and Shi, L.P. (2013) Behind the Magical Numbers: Hierarchical Chunking and the Human Working Memory Capacity. International Journal of Neural Systems, 23, Article ID: 1350019. http://dx.doi.org/10.1142/S0129065713500196

[36] Li, G.Q., Wen, C.Y., Li, Z.G., Zhang, A.M., Yang, F. and Mao, K.Z. (2013) Model-Based Online Learning with Kernels. IEEE Transactions on Neural Networks and Learning Systems, 24, 356-369. http://dx.doi.org/10.1109/TNNLS.2012.2229293 
Scientific Research Publishing (SCIRP) is one of the largest Open Access journal publishers. It is currently publishing more than 200 open access, online, peer-reviewed journals covering a wide range of academic disciplines. SCIRP serves the worldwide academic communities and contributes to the progress and application of science with its publication.

Other selected journals from SCIRP are listed as below. Submit your manuscript to us via either submit@scirp.org or Online Submission Portal.
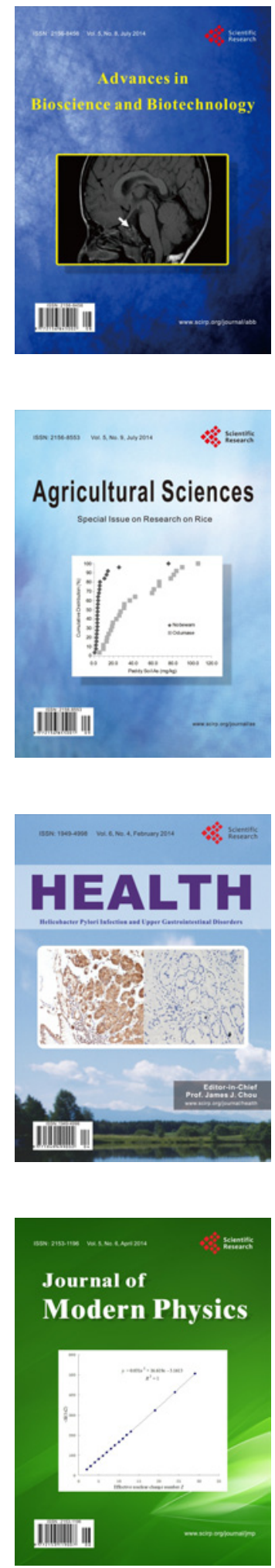
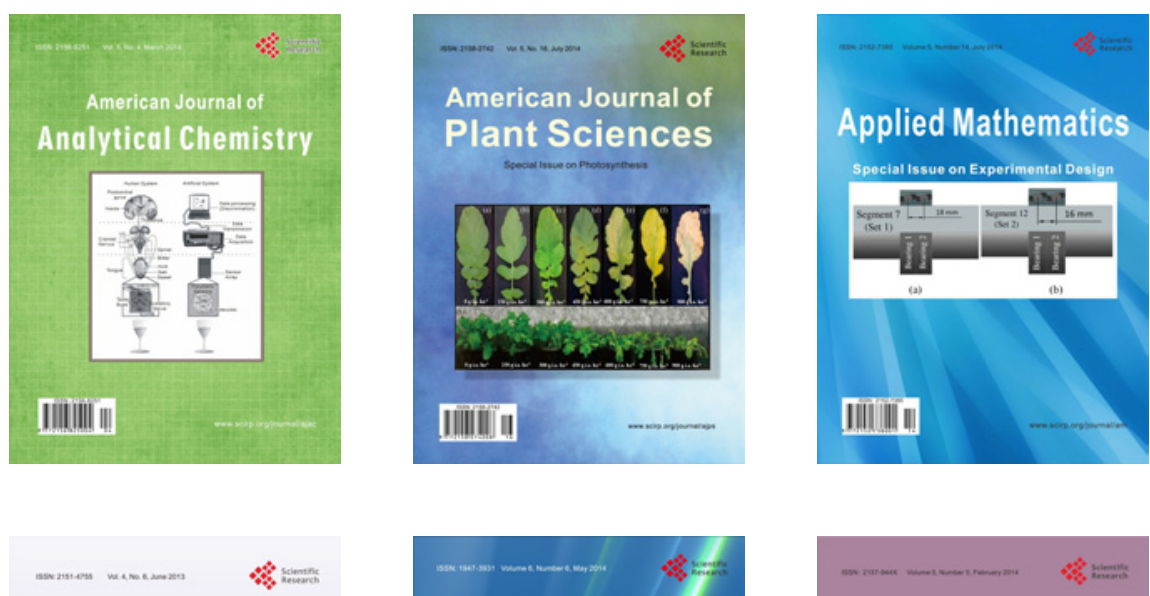

Creative Education
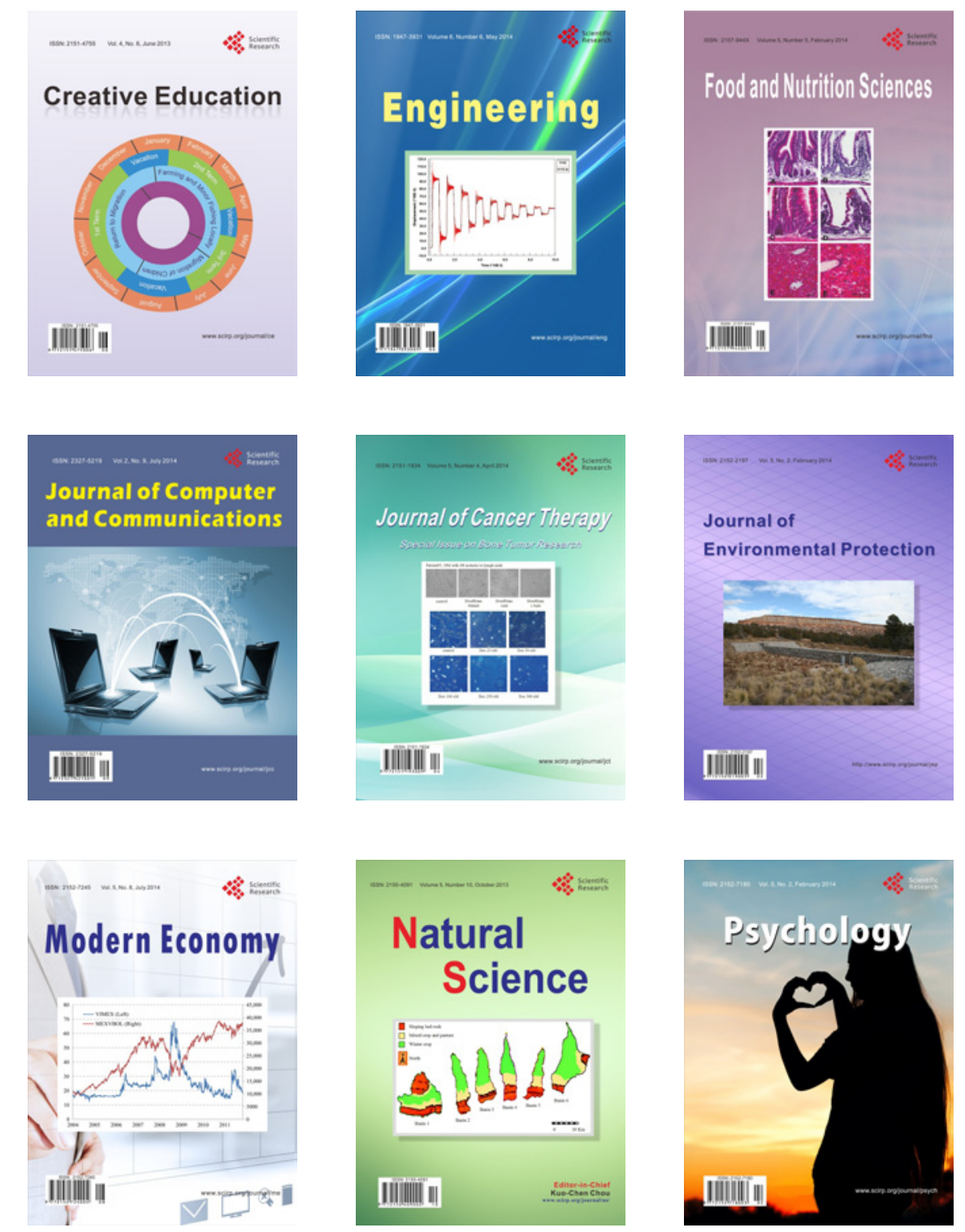